\title{
Contour Detection of Mammogram Masses Using ChanVese Model and B-Spline Approximation
}

\author{
Youssef Ben Youssef ${ }^{1}$, El hassane Abdelmounim¹, and Abdellah Lamnii * \\ ${ }^{1}$ ASTI Laboratory, ${ }^{2}$ MISI Laboratory. Hassan First University. 26000 Settat (Morocco)
}

Received 11 January 2016 | Accepted 1 November 2016 | Published 23 December 2016

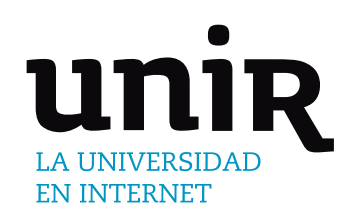

KEYWORDS

B-spline, ChanVese model, Mammogram, Segmentation.

DOI: $10.9781 /$ ijimai.2017.454

ChanVese model segmentation has been applied for contour detection of mass region in mammogram in our previous work. Available information of the desired object contour is used, in this paper, for B-spline approximation. The mass region boundary (contour) is thereafter approximated by a B-spline curve. This approach allows synthesizing the shape of the suspected mass appearing in the mammogram. Experimental results show the accurateness of mass region contour in mammograms using B-spline approximation.

\section{INTRODUCTION}

$\mathrm{B}$ REAST cancer is one of the leading causes of cancer deaths among women in the world. Early detection is a key of the reduction of women mortality. Although breast cancer incidence has increased over the last decade, breast cancer mortality has declined among women of all ages. People have the highest chance of survival if cancer could be detected at the early stages and thanks to the development of both breast cancer treatment and mammography screening. Some studies have demonstrated that mammography may be particularly beneficial for women who are 80 years of age and older $[1,2]$. According to the American Cancer society the death rate from breast cancer was increasing until 1990 when the advent of widespread screening began to have an effect on the population [3]. An abnormality detected on a mammogram is the early sign of cancer. It can appear as an abnormal area of density mass or calcification. The preferred screening examination for breast cancer is mammography. Digital mammography, and other imaging modalities such as magnetic resonance imaging (MRI), X-ray computed tomography $(\mathrm{CT})$, and ultrasound provide an effective means for non invasively mapping the anatomy of a breast. Medical images in their raw form are represented by arrays of numbers in the computer, with the numbers indicating the values of relevant physical quantities that show contrast between different types of body tissue.

Segmentation plays a significant role not only in image processing but also in pattern recognition. Medical images with high resolution and fine details are always needed and required in many visual tasks.

Image segmentation is defined as the subdividing of an image into non overlapping component regions which are homogeneous with respect to some characteristic such as intensity, color or texture $[4,5,6]$.

In digital mammography, segmentation is typically performed for detection or localization of tumors [7], micro calcification clusters [8], or other indicators of pathology [9]. In delineating suspicious masses for mammography, segmentation methods are typically employed in one

* Corresponding author.

E-mail addresses: youssefbenyoussef12@gmail.com (Youssef Ben Youssef), hassan.abdelmounim@hotmail.fr (El hassane Abdelmounim), abdellah.lamnii@uhp.ac.ma (Abdellah Lamnii). of two ways. In the first way, the mammogram is initially segmented into candidate regions which are then labeled as being suspicious or normal [10]. In the second way, the image is first processed to detect for the presence of pathology and segmentation is performed as a final step to determine its precise location $[11,12]$.

Contour or shape extraction is a very important feature in medical image processing. Many medical image analysis applications, like the measurement of anatomical structures, require prior extraction of the organ from the surrounding tissue.

In this paper, our special interest is the contour extraction of the mammogram that contains masses or tumors. The mass can be benign or malignant. The contour points extracted with Chan Vese model are used as control points for B-spline curve for getting a better contour result. The structure of this paper is arranged as follows. Section 2, briefly introduces ChanVese model for segmentation. In Section 3, a background of B-spline curve is presented. The simulation results and discussion are shown in Section 4. This paper is concluded in Section 5.

\section{Chan Vese Model Segmentation}

Analysis and processing of medical images are useful in transforming raw images into a quantifiable symbolic form for ease of searching and mining, in extracting meaningful quantitative information to aid diagnosis and make decision. One fundamental problem in medical image analysis is image segmentation, which identifies the boundaries of objects.

Classical approaches to solve segmentation are divided into different models: discontinuity approach, similarity approach, and variational methods [4]. In medical images, active contour or snakes are used heavily for boundary delineation or shape detection. Snake is defined as an energy minimization whose energy depends on its shape and location within the image. Shape of the snake is controlled by the internal forces and external forces. The external force guides the snake towards the features in the image, and internal force acts as smoothing constraint for the snake. The deformable model or snake offers several options for medical image analysis, especially mammographic images, delineation of suspect region and assist radiologists in locating potentially cancerous cases [13].

When object contour is not clearly defined as mass region in mammographic image region based model is more suitable such as Chan Vese model. CV model is a based on global and statistical 
information to overcome the inhomogeneous intensity distribution, and thus, to drive the evolving curve towards the true boundaries. Initially, Chan Vese model aims to divide image into two parts inside $\Omega_{\text {int }}$ and outside $\Omega_{\text {ext }}$ of contour $\Gamma$. The goal of the segmentation algorithm will be to minimize this energy function for a given image defined [14].

$$
\begin{aligned}
& E\left(\Gamma, c_{1}, c_{2}\right)=\lambda_{1} \int_{\Omega_{\text {int }}}\left|I_{0}(x, y)-c_{1}\right|^{2} d x d y+ \\
& \lambda_{2} \int_{\Omega_{e x t}}\left|I_{0}(x, y)-c_{2}\right|^{2} d x d y+v|\Gamma|
\end{aligned}
$$

where $\lambda_{1} \geq 0, \lambda_{2} \geq 0$, and $v \geq 0$, are fixed weight parameters, $\Gamma$ is the evolving contour, $|\Gamma|$ is its length, and $c_{1}$ and $c_{2}$ are two constants that approximate the image intensity in inside $\Gamma$ and outside $\Gamma$. This model is not based on the gradient of the image $I_{0}(x, y)$ for the stopping process. In our previous work, we have used CV model for mass delineation contour and extracted some shape descriptors [15]. In this work, we are interested to segment a single part (mass) from the whole image or background tissue.

\section{B-Spline Curve Representation and Approximation}

\section{A. Motivation}

B-splines descend from a larger family of functions named piecewise polynomial interpolation functions. In reference [16], authors study the use of continuous B-spline representation for signal processing applications such as interpolation, differentiation, filtering, noise reduction, and data compression. Among other polynomial interpolators, B-splines have the capacity to give an approximated representation of the signal [17]. Splines are useful in computer vision because they allow accurate, manipulable internal models of complex shapes [18].

The B-spline has the following important properties: i) it is a piecewise polynomial curve with a given degree. This property makes with lower degree polynomials to design a complex curve using multiple segments joined with certain continuity constraints, ii) The B-spline curve is contained in the convex hull of its control polyline defined by the B-spline control points and it can be used to represent the shape of the curve, iii) Changing of the position of the control points only locally affects the curve, this property allows to B-spline curve the flexible controllability, 4i) No straight line intersects the B-spline curve more times than it intersects the curve's control polyline, 5i) affine transformation such as rotation, translation, and scaling can be applied to the B-spline control points quite easily instead of to the curve itself. This results in the affine invariance property [19-20].

In this work, we are interested to B-spline curves which play a central role and are widely used in computer aided design. All those properties hold for the B-spline curves and play an integral part in image shape modeling for our work.

\section{B. Basic Theory}

In this section, we briefly give an explicit representation of B-spline curve. The B-spline curve $\mathrm{C}(\mathrm{u})$ of order $\mathrm{k}$,

$$
\begin{aligned}
& (1<k \leq n+1), \text { is defined by } \\
& C(u)=\sum_{i=0}^{n} P_{i} B_{i}^{k}(u) \quad u \in\left[u_{k-1}, u_{n+1}\right]
\end{aligned}
$$

where :

- $\mathrm{P}_{\mathrm{i}}, \mathrm{i}=0, \ldots, \mathrm{n}$ are called control points or de Boor points.

- $\mathrm{k}$ is the order of the polynomial segments of the B-spline curve. Order $\mathrm{k}$ means that the curve is made up of piecewise polynomial segments of degree $\mathrm{k}-1$.

- The $\mathrm{B}_{\mathrm{i}}^{\mathrm{k}}(\mathrm{u})$ are the "normalized B-spline basis functions". They are described by the order $\mathrm{k}$ and by a non-decreasing sequence of real numbers $u_{0}, u_{i}, \ldots, u_{n+k}$ called the "knot sequence". The $\mathrm{B}_{\mathrm{i}}^{\mathrm{k}}(\mathrm{u})$ is defined recursively as follows:

$$
\begin{aligned}
B_{i}^{0}(u) & = \begin{cases}=1 \text { if } & u_{i} \leq u \leq u_{i+1} \\
=0 & \text { otherwis }\end{cases} \\
B_{i}^{k}(u) & =\frac{u-u_{i}}{u_{i+k}-u_{i}} B_{i}^{k-1}(u)+\frac{u_{i+k+1}-u}{u_{i+p+1}-u_{i+1}} B_{i+1}^{k-1}(u)
\end{aligned}
$$

The curve $\mathrm{C}(\mathrm{u})$ possess the following properties analogous to those of the Bézier curves:

- Endpoint interpolation property

- Convex hull property: the basis functions have the properties of non-negativity and partition of unity, as a consequence, the entire B-splines curve segment must lie inside the control polygon spanned by $\mathrm{P}_{0}, \ldots, \mathrm{P}_{\mathrm{n}}$.

- Variation diminishing property : no straight line intersects a B-splines curve more times than it intersects its control polygon.

- Convexity-preserving property: the variation diminishing property means the convexity preserving property holds.

\section{RESUlTS AND Discussion}

\section{A. Methodology}

In order to evaluate our method, we have used image mammogram extracted from MIAS database [22]. The Mammographic Image Analysis Society (MIAS) MIAS is an organization of research groups on mammograms of the United Kingdom (UK). The mammogram, in original MIAS database, is digitized at 50 micron pixel edge and has been reduced to 200 micron pixel edge padded so that every image is 1024 x1024 pixels. All images are held as 8-bit gray level scale images with 256 different gray levels (0-255) and physically in portable gray map (pgm) format. For each abnormal case (benign and malign), MIAS database gives the coordinates of abnormality center and the approximate radius of a circle enclosing this abnormality; it contains 322 images, where 207 images represent normal, while 64 and 51 images referred as benign and malignant cases respectively. The MIAS database is limited on single view mediolateral oblique.

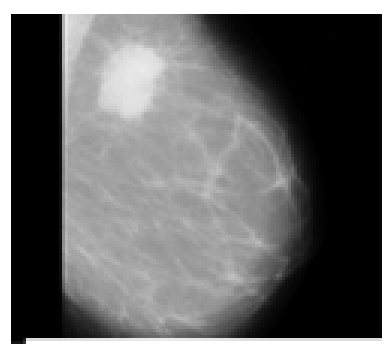

(a)

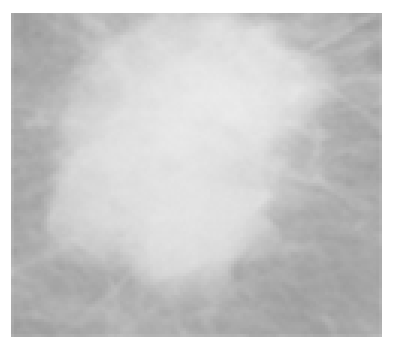

(b)
Fig. 1. (a) Original image, (b) image cropped and resized.

Original image from mammogram data is depicted in Fig.1(a), and image obtained after cropping and resizing preprocessing is shown in Fig.1(b) which contains a mass called region of interest (ROI). This technique is needed for reducing cost computing and removing the unnecessary data.

\section{B. Contour Extraction using CV Model and B-Spline Curve Approximation}

After cropping and resizing mammographic image where mass is isolated, we use ChanVese model for segmentation. Mass contour points are used as control points. Then B-splines curve is used for contour fitting. The results obtained are shown in Fig. 2. 


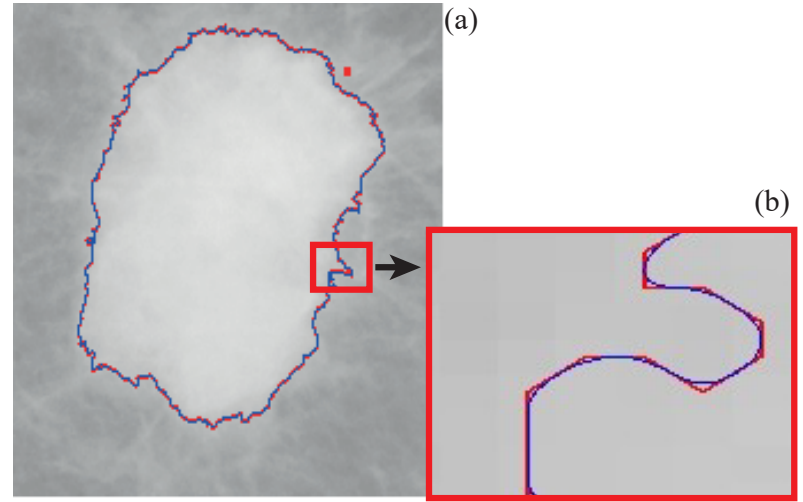

Fig 2. (a) Mass region segmented. (b) Zoom-in view of the region inside the square.

Fig. 2(a) presents a mammogram (mdb184) where a circumscribed mass is segmented with CV model (red curve). While the B-spline curve used to fit the contour is represented in blue. Fig. 2(b) shows the B-spline approximation of a given 10 control points.

It can be seen from Fig.2(b) the difference betwen contour obtained with ChanVese model and B- spline curve approximation. As shown in the zooming part of the contour, it is noted that the contour provided by B-spline is a complementary processing of the contour detection provided by $\mathrm{CV}$ model which give a realistic contour rather than a curve line represented by a series of juxtaposed segments presented as In the approximation, the B-spline curve does not have to pass through all data points except the first and the last data points.

\section{Conclusion}

In this work, we have used the ChanVese model for mammogram segmentation, in which the local image information is incorporated. CV model is an efficient and accurate method to isolate and extract masses in mammograms, and is better adapted to perform segmentation of regions with weak boundaries and noise. The data points used in this work are obtained from contours of the segmented regions after performing mammogram segmentation. These contour points extracted are used as control points for B-spline curve to give a realistic contour of the ROI.

\section{REFERENCES}

[1] MA. Schonberg , RA. Ramanan , EP..McCarthy, ER.Marcantonio "Decision making and counseling around mammography screening for women aged 80 or older". J Gen Intern Med. 21 (9), pp. 979-85, September 2006.

[2] B.D.Badgwell, S.H.Giordano, Z.Z.Duan, S.Fang,I.Bedrosian, H.M.Kuerer, "Mammography Before Diagnosis Among Women Age 80 Years and Older With Breast Cancer" J Clin Oncol. April 2008.

[3] American Cancer Society. Breast Cancer Facts \& Figures 2013-2014. American Cancer Society. Available at http://www.cancer.org/acs/groups/ content/@research/documents/document/acspc-042725.pdf. Accessed: May 6, 2015.

[4] R.C. Gonzalez and R.E. Woods, Digital Image Processing. AddisonWesley, 1992.

[5] R.M. Haralick and L.G. Shapiro. "Image segmentation techniques. Comput”. Vis. Graph. Im. Proc.,29, pp. 100-132, 1985.

[6] R.J. Schalkoff. Pattern recognition: statistical, structural and neural approaches. John Wiley and Sons, 1992.

[7] S. Pohlman, K.A. Powell, N.A. Obuchowski, W.A. Chilcote, and S. Grund fest-Broniatowski. "Quantitative classification of breast tumores in digitzed mammograms” Med. Phys., 2, pp.1337-1345, 1996.

[8] H. Cheng, Y.M. Lui, and R.I. Freimanis, "A novel approach to microcalcification detection using fuzzy logic technique", IEEE Tran. Med. Imag., 17, pp.442-450, 1998.

[9] M. Kallergi, K. Woods, L.P. Clarke, W. Qian, and R.A. Clark,'Image segmentation in digital mammography: comparison of local thresholding and region growing algorithms", Comput. Med. Im. Graph., 16, pp.323-331, 1992.

[10] H.D. Li,M. Kallergi, L.P. Clarke, V.K. Jain, and R.A. Clark, "Markov random field for tumor detection in digital mammography". IEEE Trans. Med. Imag., 14, pp.565-576, 1995.

[11] R. Gupta and P.E. Undrill,"The use of texture analysis to delineate suspicious masses in mammography", Phys. Med. Biol., 40, pp.835-855, 1995.

[12] C.H. Chen and G.G. Lee,"On digital mammogram segmentation and microcalcification detection using multiresolution wavelet analysis" Graph. Mod. Im. Proc., 59, pp. 349-364, 1997.

[13] Y.BenYoussef, E.H.Abdelmounim, J. Zbitou. and A, Belaguid, "Segmentation of mass region in abnormal mammogram using deformable Model" .International Journal of Emerging Technology and Advanced Engineering.vol 4 (7), pp.578-582, 2014.

[14] T.F.Chan, and L.A. Vese, "Active contours without edges".IEEE transaction on image processing, (10), pp. 266-277. 2001.

[15] Y. BenYoussef, E. Abelmounim, J. Zbitou and A.Belaguid," Global and Local Descriptors Comparison for Classification into Malignant and Benign Mammograms using Support Vector Machine”, Mediteranean Conference on Information \& Communication Technologies'2015 May 7-9, 2015 Saïdia Morocco.

[16] M, Unser,A. Aldroubi,and M.Edden,'B-Spline signal processing:Part I-Theory", IEEE Trans. Sign. Proces., Vo41,2,February, 1993.

[17] C. Ciulla," Improved Signal and Image Interpolation in Biomedical Applications: The Case of Magnetic Resonance Imaging (MRI)", Medical Information science reference, Hershy New York, 2009.

[18] A.Blake and M.Isard, “Active Contours". Springer Verlag. 1998.

[19] F.Mokhtarian, and A.K.Mackworth, "A theory of multiscale, curvaturebased shape representation for planar curves", Pattern Analysis and Machine Intelligence, IEEE Transactions on, Vol. 14, Issue 8, pp. 789805, Aug. 1992.

[20] D.H. Ballard, C.M, Brown, "Computer Vision”,Prentice Hall,1982.

[21] P. Saint-Marc, H. Rom, and G. Medioni, "B-spline contour representation and symmetry detection," in Pattern Analysis and Machine Intelligence, IEEE Transactions on, vol.15, no.11, pp.1191-1197, Nov 1993.

[22] J.Suckling, J,D.Parker, S.Dance, I,Astley, I.Hutt, and C. Boggis.”The mammographic image Analysis society digital mammogram database", exerpta medica. International congress seriesvol(1069),375-378,1994.

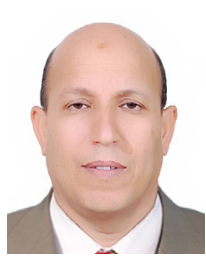

\section{Youssef Benyoussef}

Youssef Benyoussef is currently pursuing his PhD Degree in System Analysis and Information Technology Laboratory "ATSI" in science and technology faculty Hassan 1st, University Settat Morocco. He holds a Bachelor in electronic at Mohammed 1st university Oujda in 1993, and Master of Science and Technology in electrical engineering at Hassan 1st university in 2011. His research interests are on computer vision, image processing, machine learning, and artificial intelligence. In 1995, he graduated from the aggregation in physics from Higher Normal School. Currently, he is professor of physics in preparatory class for high schools, Settat, Morocco.

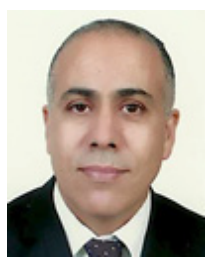

Elhassane Abdelmounim

Elhassane Abdelmounim was born in Oued-Zem Morocco in 1965, he received his $\mathrm{PhD}$ in Spectral analysis from Limoges University at Science and Technology Faculty, France in 1994. In 1996, he joined, as Professor, applied physics department of Science and Technology faculty, Hassan 1st University, Settat, Morocco. His current research interests include digital signal processing and machine learning. He is currently coordinator of a Bachelor of Science in electrical engineering and he is researcher at System Analysis and Information Technology Laboratory at Hassan 1st University, Settat, Morocco.

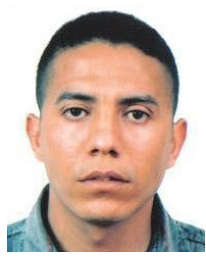

Abdellah Lamnii

Abdellah Lamnii was born in Ain Beni Mathar Morocco in 1978. PhD in applied mathematics from Mohammed 1st university Oujda-Morocco, cotutelle with the University of Pau Et pays de l'adour in 2009. 2010 as Professor, applied mathematics Department of Mathematics and Informatics, Hassan 1st University, Settat, Morocco. His current research interests include applied mathematics and digital signal. 